\title{
Correspondence
}

\section{ATG5 can regulate p53 expression and activation}

\author{
HU Simon ${ }^{\star, 1}$, S Yousefi ${ }^{1}$ I Schmid ${ }^{1}$ and R Friis ${ }^{1}$ \\ Cell Death and Disease (2014) 5, e1339; doi:10.1038/cddis.2014.308; published online 17 July 2014
}

\section{Dear Editor,}

DNA-damaging anti-cancer drugs cause cell death by apoptosis, but they also activate macroautophagy ${ }^{1}$ (hereafter just autophagy), a fundamental survival pathway under stress where cells enclose cytosol or organelles in double-membrane autophagosomes, then fuse them with lysosomes for recovery of metabolic precursors. ${ }^{2}$ This process depends upon autophagy-related (ATG) proteins. $^{2}$ Activation of this survival pathway is unwanted in cancer therapy, because even a few surviving tumor cells can accumulate mutations, gain genetic diversity, and, potentially, resume proliferation. $^{3}$

We recently reported that expression of ATG5 was upregulated by treatment with low concentrations of etoposide. ${ }^{4}$ Few cells entered apoptosis, but almost all showed autophagy. Surprisingly, much of the induced ATG5 was found in the cell nucleus, binding to BIRC5/survivin and causing cell cycle arrest at $\mathrm{G}_{2} / \mathrm{M}$ followed by mitotic catastrophe. $^{4,5}$ We then asked whether just ectopic ATG5 expression, without etoposide, would lead to mitotic catastrophe. Indeed, a large part of the expressed ATG5 was again found in the nucleus and rapid cell cycle arrest together with mitotic catastrophe was observed. ${ }^{4}$ DNA damage, however, was not in evidence; neither ATM nor ATR phosphorylation was detected and foci of $\mathrm{H} 2 \mathrm{AX}$ phosphorylation were absent. ${ }^{4}$ This has led us to ask: how can ATG5 induce the same kind of stress response as DNA-damaging drugs?

This report documents increased p53 expression after lentivirus-mediated, ectopic ATG5 expression (Figure 1a). Upregulated p21 demonstrated p53 transactivation of a target gene. Vector alone elicited only a slightly elevated p53. As expected, ATG5 expression also caused autophagy as measured by lipidated LC3 (LC3-II).

One wonders whether the observed p53 upregulation/ activation is necessary to initiate autophagy? ${ }^{6}$ Using p53 null Saos- 2 cells made DOX inducible for p53 with a Tet-on construct, we showed that p53 was not required for autophagy (Figure 1b). Ectopically expressed ATG5 is shown as both 33-kDa monomer and 57-kDa conjugate with ATG12. Autophagy was apparent from LC3-II. An additional stimulus with nutrient starvation was followed within $1 \mathrm{~h}$ by further increased LC3-II, but without any requirement for p53 induction (Figure 1b).

As lentivirus-mediated gene transfer might have produced some local anomalies in DNA, we studied p53 expression/ activation in an ATG5 knockout mouse embryo fibroblast (mEF) line that had been subsequently transfected with a Tet-off ATG5 expression system (clone M5-7). ${ }^{7}$ As these cells had been maintained since 2006 without DOX stimulation, a DNA damage response seems unlikely; however, we examined ATM phospho-Ser1981 as confirmation (Figure 1c). After DOX treatment, ATG5 expression in these cells was rapidly suppressed. Note: please ignore the unspecific band seen in mEF cells at $36 \mathrm{kDa}$. In mEF cells, ATG5 exists almost entirely as a conjugate with ATG12. Corresponding to the downregulation of ATG5 after DOX, both p53 expression and autophagy, monitored as LC3-II levels, declined sharply (Figure 1c). Also in Figure 1d, using the same Tet-off ATG5 expression system, these differences between ATG5-non-expressing and ATG5-expressing cells were apparent. Noteworthy is that, ATG5-expressing cells showed not only elevated p53 levels, but also increased p53 activation as evidenced by p53 Ser18 phosphorylation (Figure 1d). Interestingly, starvation also induced p53 in DOX-treated cells not expressing ATG5, not, however, LC3-II or autophagy.

Our findings thus indicate that increased ATG5 expression represents for the cell something like a stress response. In consequence, p53 upregulation and transactivation of p21 are followed by cell cycle arrest. Here p53 is not acting in its generally accepted role as a regulator of autophagy and apoptosis, ${ }^{6}$ but itself exhibits a secondary response to upregulation of ATG5 expression. Similar findings have been reported recently with $A T G 7$ knockout mEF cells, ${ }^{8}$ showing that ATG7 also can impose a reciprocal regulation on p53. Surprisingly too, activation of the NF- $\kappa \mathrm{B}$ pathway, an important stress response, is blocked in cells lacking either ATG5 or ATG7. ${ }^{9}$ All these observations suggest a network of interactive responses available to the cell, initiated by different homeostatic imbalances, but integrated in the same overall program.

\footnotetext{
${ }^{1}$ Institute for Pharmacology, University of Bern, Friedbuehlstr. 49, CH-3010 Bern, Switzerland

${ }^{*}$ Corresponding author: HU Simon, Institute for Pharmacology, University of Bern, Friedbuehlstr. 49, CH-3010 Bern, Switzerland. Tel: +41 316323281 ; Fax: +41 31632 4992; E-mail: hus@pki.unibe.ch
} 

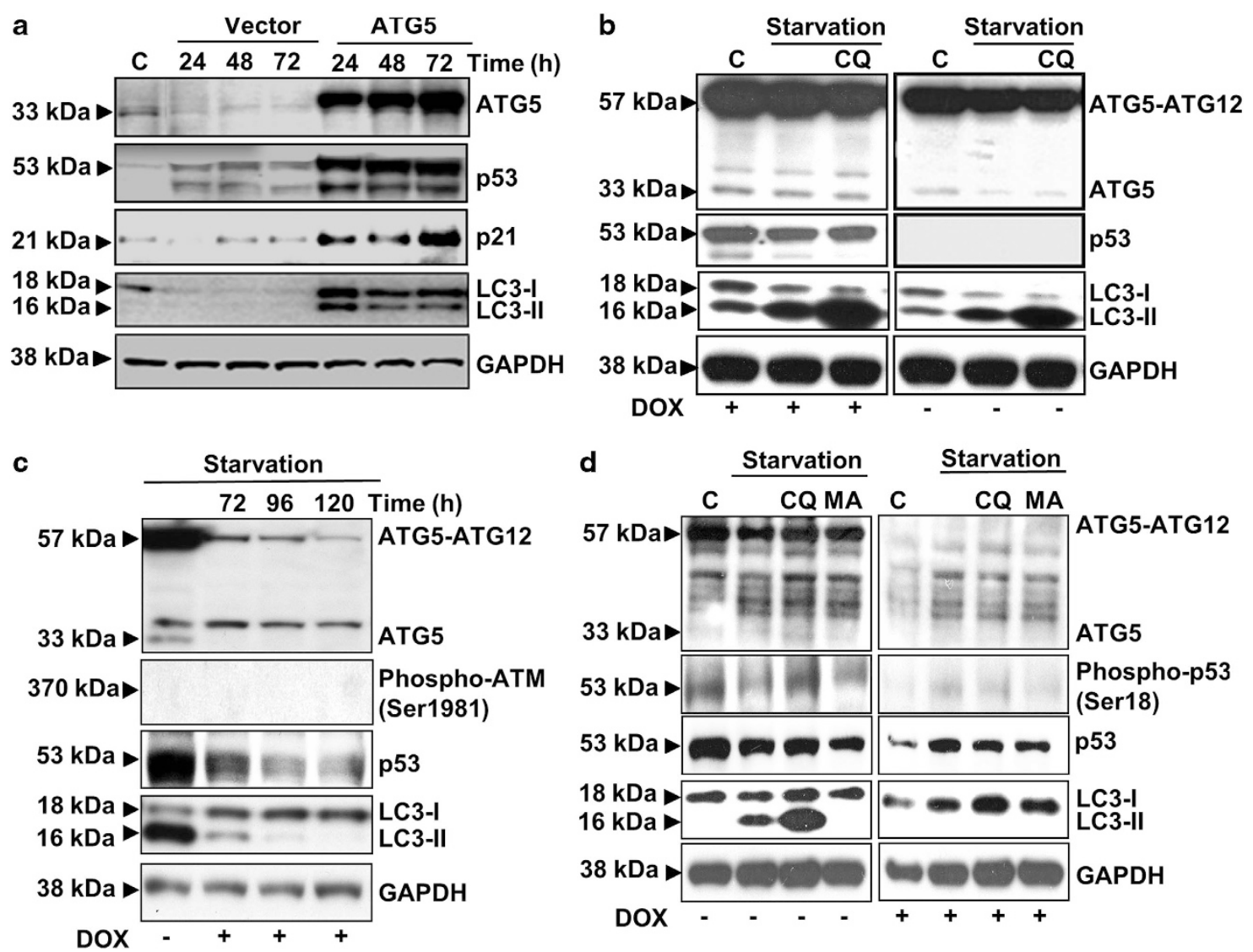

Figure 1 ATG5 expression induces and activates p53. (a) Jurkat T cells were transduced with lentivirus constructs of vector alone or ATG5. Here shown is the ATG5 33-kDa monomer band. At the indicated intervals in hours, lysates were prepared, electrophoresed and processed for immunoblotting with the respective antibodies as shown to the right of each panel. Molecular weights are indicated on the left. GAPDH served as a loading control. ' $C$ ' designates an untransduced control. (b) Saos-2 cells, DOX inducible for p53, were all transduced with the ATG5 lentivirus construct. After $24 \mathrm{~h}$, half the cultures received DOX and all were incubated a further $24 \mathrm{~h}$. Control cultures remained in standard medium, whereas all others were starved for the final hour in amino acid-free medium before being processed for immunoblotting as in a. Chloroquine (CQ) was used as a control for the effect of blocked lysosomal function. (c) The M5-7 clone of mEF cells suppressed ATG5 production upon DOX treatment. Cultures were untreated or DOX-treated for the indicated intervals and, in all samples, subjected to amino-acid starvation during the final hour before lysis and processing for immunoblotting. (d) M5-7 cells were untreated or treated with DOX for $120 \mathrm{~h}$. In each group, one control remained in standard medium; all others were starved for the final hour before lysis and processing for immunoblotting. $C Q$ and 3-methyl adenine (MA) were employed as controls. All data are representative for at least three independent experiments

\section{Conflict of Interest}

The authors declare no conflict of interest.

Acknowledgements. We thank Drs Nao Hosokawa and Noboru Mizushima (Tokyo Metropolitan Institute of Medical Science) for providing us with the Tet-off ATG5 M5-7 mouse embryo fibroblast clone and Dr Gerry Melino (University of Rome, 'Tor Vergata') for the Saos-2 p53 inducible-expression clone.

1. Rodriguez-Rocha $\mathrm{H}$ et al. Mutat Res 2011; 711: 158-166.

2. Yang Z, Klionsky DJ. Curr Opin Cell Biol 2010; 22: 124-131.

3. Vitale I et al. Cell Death Differ 2011; 18: 1403-1413.

4. Maskey D et al. Nat Commun 2013; 4: 2130.

5. Simon HU, Friis R. Autophagy 2013; 10: 176-177.

6. Pietrocola F et al. Sem Cancer Biol 2013; 23: 310-322.

7. Hosokawa $\mathrm{N}$ et al. FEBS Letters 2006; 580: 2623-2629.
8. Lee IH et al. Science 2012; 336: 225-228.

9. Criollo A et al. Cell Cycle 2012; 11: 194-199. (c) (i) $(-)$ Cell Death and Disease is an open-access journal published by Nature Publishing Group. This work is licensed under a Creative Commons Attribution-NonCommercialNoDerivs 3.0 Unported License. The images or other third party material in this article are included in the article's Creative Commons license, unless indicated otherwise in the credit line; if the material is not included under the Creative Commons license, users will need to obtain permission from the license holder to reproduce the material. To view a copy of this license, visit http://creativecommons.org/licenses/ by-nc-nd/3.0/ 